\title{
EFFECT OF ATTENUATED MP12 RIFT VALLEY FEVER VACCINE ON IMMUNE RESPONSE OF SHEEP
}

\author{
SAYED, TARADI A. ${ }^{1}$, A. M. IBRAHIM ${ }^{1}$, LILY S. SALAMA ${ }^{1}$, T. N. MARCOS $^{1}$ \\ AND A. K. SOLIMAN ${ }^{2}$ \\ 1 Veterinary Serum and Vaccine Research Institute, Ministry of Agriculture, ARC, \\ Dokki, Giza, Egypt \\ 2 NAMRU - Cairo
}

(Manuscript received 30 March 2011)

\begin{abstract}
This work aimed to study the clinical aspects and immune response of pregnant and non-pregnant ewes vaccinated with attenuated MP12 RVF vaccine. Non-pregnant ewes showed slight elevation of body temperature lasted for one day post-vaccination. There were no detectable clinical signs of RVF disease. Pregnant ewes showed no elevation in body temperature, no abortion and delivered healthy lambs (without abnormalities). RVF virus was detected on the $2^{\text {nd }}$ day post-vaccination in 15 out of 18 serum samples using RT- PCR. IgM antibodies were detected on 7 days post-vaccination (DPV) and persisted up to 150 DPV with a titer ranged from $100-800$. IgG antibodies were detected on 7 DPV reaching the peak on 60 DPV and persisted up to 360 DPV with a titer ranged from $100-800$. Maternal immunity appeared in newly born lambs after ingestion of colosrum and lasted up to 4 months. It is concluded that MP12 RVF vaccine is safe and potent for vaccination of sheep.
\end{abstract}

\section{INTRODUCTION}

Rift Valley Fever (RVF) is a peracute or acute, febrile mosquito born zoonotic disease caused by a virus of the family Bunyaviridae, genus Phlebovirus. The disease is characterized by high rate of abortion and neonatal mortality primarily in sheep, goat and cattle (OIE, 2008). Natural occurrences of RVF were first documented in Egypt in 1978, where killed more than 600 people and caused sickness to over 200,000 (Meegan, 1979), and another outbreak in 1993 (MMWR, 1994). An outbreak in Yemen in 2000 was the first documented occurrence outside Africa (MMWR, 2000).

Vaccine development is focusing on protecting both animals and humans. One of the most promising is the live attenuated vaccine candidate MP-12. This vaccine was developed by researchers at the US Army Medical Research Institute of Infectious Diseases (USAMRIID). The vaccine has proved efficacy and safety in sheep and cattle (Caplen, et. al., 1985). One dose of attenuated virus vaccine proved long term immunity, but not better in pregnant animals. The inactivated virus vaccine doesn't have side effect, but multiple doses were given to provide protection (WHO, 2007). 
Immunoglobulin - M (IgM) could be detected through out the period from the $7^{\text {th }}$ day up to $21^{\text {st }}$ day post-vaccination, and persisted for a short duration when the live attenuated RVF vaccine was used, but was not detectible in case of inactivated vaccine (Elian and Botros, 1997), while immunoglobulins - G (IgG) lasted for 36 months post-vaccination with live attenuated smithburn vaccine (Hassan, et al, 2001).

ELISA was reported as safe, robust, and highly accurate technique used in early diagnosis of infection, disease surveillance and for monitoring of immune response in vaccines (Paweska, et. al., 2005).

\section{This work aimed to:}

1. Evaluate the clinical aspects of the mutagenized Rift Valley Fever MP12 vaccine in pregnant and non-pregnant local sheep breed.

2. Assess the post-vaccination immune response (IgG \& IgM) and duration of immunity.

3. Assess the safety of the MP12 vaccine (abortoginicity \& teratogenicity) during the study.

\section{MATERIALS AND METHODS}

Twenty-one Balady sheep (less than 3 years old) were divided into three groups, first one (G1) (14 non-pregnant ewes) and $2^{\text {nd }}$ one (G2) (4 pregnant ewes at $1^{\text {st }}$ trimester) were vaccinated with $10^{5} \mathrm{PFU}$ attenuated MP12 vaccine, while, the $3^{\text {rd }}$ one (control) (3 sheep) was kept as non-vaccinated negative control. All groups were examined daily for any clinical signs, and rectal temperatures were recorded till 6 days post-vaccination (DPV). Sheep of pregnant group were observed till parturition and nursing.

Lyophilized RVF-ZH548-MP12 mutagenized vaccine was supplied by NAMRU 3. This vaccine was developed by workers at the US army Medical Research Institute for Infectious Diseases (USAMRIID) by serially passaging of a human virus isolate (strain ZH548) in human diploid fibroblast cells in the presence of the mutagen 5fluorouracil (Caplen et. al.,1985).

Sheep sera samples were collected from all groups on $0,2,7,14,28,60$, 120, 150, 210 and 360 DPV. Lamb sera samples were collected before colosrum ingestion and 2, 30, 60, 90 and 120 days after colosrum ingestion.

Determination of immunoglobulin (IgG) and (IgM) against RVFV was carried out using ELISA technique (titer $\leq 1: 50$ consider positive) according to OIE(1989). 
RT - PCR for detection of RVF RNA was done according to Sall et. al. (2002) on sera samples from vaccinated sheep on zero and $2^{\text {nd }}$ DPV.

\section{RESULTS AND DISCUSSION}

Table 1. Temperature and clinical signs of sheep vaccinated with MP12 RVF vaccine.

\begin{tabular}{|c|c|c|c|c|c|c|c|c|c|c|c|c|}
\hline & & & & & Temp & ture ( & C) result & and cli & ical sic & & & \\
\hline & & Anima & & & & Day & post-vacc & ation & & & & \\
\hline & & I No. & ze & & & & 2 & & 3 & 4 & 5 & 6 \\
\hline & & & $M$ & $E$ & $M$ & $E$ & $M$ & $E$ & $M$ & $M$ & $M$ & $M$ \\
\hline & & 1 & $39-$ & 39.7 & 39.3 & 39.7 & $39.2+$ & 39.2 & 39 & 39.3 & 38.4 & 38.2 \\
\hline & & 2 & $38.8-$ & 39.5 & 39.4 & 39.3 & $39.4+$ & 39.2 & 39.7 & 39.5 & 38.7 & 38.0 \\
\hline & $\stackrel{0}{\not}$ & 3 & $38.9-$ & 39.5 & 38.4 & 39.2 & $39-$ & 39.4 & 39.7 & 38.4 & 38.6 & 38.2 \\
\hline & d & 4 & $39-$ & 39.3 & 39.5 & 40 & $40+$ & 39.8 & 39.2 & 38.4 & 38 & 38.1 \\
\hline & 苾 & 5 & $39-$ & 39.5 & 39.5 & 39.5 & $39+$ & 39.1 & 39.9 & 38.7 & 38.2 & 38.7 \\
\hline సే & 鿒 & 6 & $38.9-$ & 39.7 & 41 & 40.8 & $39.4+$ & 39.9 & 39.8 & 38.6 & 38.2 & 38.5 \\
\hline & 这 & 7 & $39-$ & 39.5 & 39.8 & 40.5 & $39.1-$ & 39.5 & 39.3 & 38.7 & 38.3 & 38.7 \\
\hline & 운 & 8 & $38.9-$ & 39.6 & 39.3 & 39.8 & $39.9+$ & 39.3 & 39.5 & 38.5 & 39.0 & 38.5 \\
\hline & & 9 & $38.8-$ & 39.2 & 40.2 & 40.1 & $39+$ & 39.2 & 38.4 & 38.4 & 38.3 & 38.9 \\
\hline & & 10 & $38.6-$ & 39.7 & 40.3 & 40.1 & $39.5+$ & 39.7 & 38.7 & 39 & 38.5 & 38.0 \\
\hline & & 11 & $39-$ & 39.3 & 39.8 & 39.9 & $39+$ & 39.7 & 38.5 & 38.5 & 38.4 & 39.1 \\
\hline & & 12 & $39-$ & 39.5 & $40.5 \mathrm{D}$ & $40.2 \mathrm{D}$ & $\begin{array}{c}39.5 \\
D+\end{array}$ & 39.2 & 38.4 & 39.7 & 39 & 39.0 \\
\hline & & 13 & $38.9-$ & 39.8 & 40.2 & 40.1 & $39.1+$ & 39.9 & 38.4 & 39.7 & 38.4 & 39.0 \\
\hline & & 14 & $39-$ & 39.8 & 40.9 & 40.8 & $39.3+$ & 39.8 & 38.7 & 39.2 & 38.7 & 39.0 \\
\hline & & 1 & $38.6-$ & 39.8 & 38.9 & 39.5 & $38.8-$ & 39.7 & 38.6 & 39.9 & 39.0 & 38.6 \\
\hline$\widetilde{N}$ & $\bar{G}+\frac{8}{0}$ & 2 & $39-$ & 39.7 & 39.3 & 39.6 & $39.3+$ & 39.7 & 38.7 & 39.8 & 38.7 & 38.3 \\
\hline & & 3 & $38.9-$ & 39.7 & 39.6 & 39.6 & $39.2+$ & 39.3 & 38.5 & 38.4 & 38.5 & 39.1 \\
\hline & & 4 & $38.6-$ & 39 & 39.5 & 39.2 & $38.5+$ & 39.5 & 38.4 & 38.7 & 38.4 & 39.0 \\
\hline & Non- & 1 & $38.5-$ & 38.4 & 38.6 & 38.5 & 38.7 & 38.4 & 38.4 & 38.7 & 38.6 & 38.6 \\
\hline & & 2 & $38.7-$ & 38.7 & 38.4 & 38.6 & 38.5 & 38.4 & 38.7 & 38.5 & 38.4 & 38.6 \\
\hline & & 3 & $38.6-$ & 38.6 & 38.4 & 38.7 & 38.4 & 38.6 & 38.6 & 38.4 & 38 & 38.4 \\
\hline
\end{tabular}

Table 2. Results of immunoglobulin (IgM) titer of sheep vaccinated with MP12 RVF vaccine using ELISA technique.

\begin{tabular}{|c|c|c|c|c|c|c|c|c|}
\hline & & & & & & & & \\
\hline & & Allitild & & & ays p & cinati & & \\
\hline & & & 0 & 7 & 14 & 28 & 60 & 150 \\
\hline & & 1 & -ve & 200 & 200 & 200 & 100 & 100 \\
\hline & & 2 & -ve & 400 & 400 & 400 & 100 & 100 \\
\hline & $\sum_{z}^{\infty}$ & 3 & -ve & 100 & 200 & 100 & 100 & 100 \\
\hline & ฮ & 4 & -ve & 200 & 400 & 200 & 200 & 200 \\
\hline & 蒙 & 5 & -ve & 400 & 400 & 100 & 100 & 100 \\
\hline 厄) & 号 & 6 & -ve & 100 & 200 & 100 & 100 & 100 \\
\hline & ర్ & 7 & -ve & 100 & 100 & 100 & 100 & 100 \\
\hline & $\frac{0}{1}$ & 8 & -ve & 200 & 400 & 100 & 100 & 100 \\
\hline & 은 & 9 & -ve & 400 & 400 & 100 & 100 & 100 \\
\hline & & 10 & -ve & 100 & 200 & 100 & 100 & 100 \\
\hline & & 11 & -ve & 200 & 200 & 200 & 100 & 100 \\
\hline & & 12 & -ve & 400 & 400 & 800 & 800 & 800 \\
\hline & & 13 & -ve & 400 & 400 & 100 & 200 & 100 \\
\hline & & 14 & -ve & 100 & 100 & 100 & 100 & 100 \\
\hline & & 1 & -ve & 100 & 200 & 200 & 200 & 100 \\
\hline & 它 & 2 & -ve & 100 & 200 & 200 & 100 & 100 \\
\hline ن & 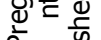 & 3 & -ve & 100 & 100 & 100 & 100 & 100 \\
\hline & & 4 & -ve & 200 & 400 & 200 & 100 & 100 \\
\hline & n- & 1 & -ve & $-\mathrm{ve}$ & -ve & -ve & -ve & -ve \\
\hline & nated & 2 & -ve & $-\mathrm{ve}$ & -ve & -ve & $-\mathrm{ve}$ & -ve \\
\hline & & 3 & -ve & -ve & -ve & -ve & -ve & -ve \\
\hline
\end{tabular}


Table 3. Results of immunoglobulin (IgG) titer of sheep vaccinated with MP12 RVF vaccine using ELISA technique.

\begin{tabular}{|c|c|c|c|c|c|c|c|c|c|c|}
\hline & & & & & & & & & & \\
\hline & & AlIIIII & & & & ys po & ccina & & & \\
\hline & & & 0 & 7 & 14 & 28 & 60 & 150 & 210 & 360 \\
\hline & & 1 & $-v e$ & 50 & 100 & 100 & 200 & 200 & 200 & 100 \\
\hline & & 2 & -ve & 50 & 100 & 400 & 400 & 200 & 100 & 100 \\
\hline & & 3 & $-v e$ & 100 & 400 & 400 & 800 & 800 & 800 & 800 \\
\hline & 亗 & 4 & $-v e$ & 100 & 400 & 400 & 400 & 400 & 400 & 200 \\
\hline & $\ddot{ت}$ & 5 & -ve & 100 & 200 & 400 & 800 & 400 & 200 & 100 \\
\hline ज゙ & 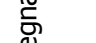 & 6 & - ve & 50 & 100 & 400 & 400 & 200 & 200 & 200 \\
\hline & $\frac{\mathrm{s}}{1}$ & 7 & $-v e$ & 50 & 100 & 400 & 200 & 200 & 200 & 200 \\
\hline & бо & 8 & -ve & 50 & 100 & 200 & 200 & 200 & 200 & 100 \\
\hline & & 9 & -ve & 100 & 200 & 200 & 200 & 200 & 200 & 100 \\
\hline & & 10 & $-v e$ & 50 & 400 & 800 & 800 & 800 & 400 & 200 \\
\hline & & 11 & -ve & 100 & 200 & 200 & 200 & 400 & 200 & 100 \\
\hline & & 12 & -ve & 50 & 100 & 200 & 200 & 200 & 200 & 100 \\
\hline & & 13 & - ve & 50 & 100 & 200 & 200 & 200 & 200 & 100 \\
\hline & & 14 & -ve & 100 & 200 & 200 & 200 & 400 & 200 & 100 \\
\hline & & 1 & $-v e$ & 50 & 100 & 200 & 400 & 400 & 200 & 100 \\
\hline$\pi$ & 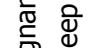 & 2 & - ve & 50 & 100 & 200 & 200 & 200 & 400 & 200 \\
\hline & & 3 & -ve & 50 & 200 & 400 & 400 & 200 & 200 & 200 \\
\hline & & 4 & -ve & 100 & 200 & 400 & 400 & 400 & 200 & 100 \\
\hline & & 1 & -ve & -ve & -ve & -ve & -ve & -ve & -ve & -ve \\
\hline & nated & 2 & $-v e$ & -ve & -ve & -ve & -ve & -ve & -ve & -ve \\
\hline & & 3 & -ve & -ve & -ve & -ve & -ve & -ve & -ve & -ve \\
\hline
\end{tabular}

Table 4. The mean titer of immunoglobulins $M$ and $G$ (IgM \& IgG) of sheep vaccinated with MP12 RVF vaccine using ELISA technique.

\begin{tabular}{|c|c|c|c|c|c|c|c|c|c|}
\hline \multicolumn{2}{|c|}{$\begin{array}{c}\text { Sheep } \\
\text { groups }\end{array}$} & \multicolumn{8}{|c|}{ Mean titer / Days post-vaccination } \\
\cline { 2 - 10 } & 0 & 7 & 14 & 28 & 60 & 150 & 210 & 360 \\
\hline \multirow{2}{*}{ IgM } & G1 & -ve & 235.7 & 285 & 192.8 & 164.7 & 157.1 & 0 & 0 \\
\cline { 2 - 10 } & G2 & -ve & 125 & 225 & 175 & 125 & 100 & 0 & 0 \\
\hline \multirow{2}{*}{ IgG } & G1 & -ve & 71.5 & 192.5 & 321.4 & 371.4 & 342.8 & 264.2 & 178.5 \\
\cline { 2 - 10 } & G2 & -ve & 62.5 & 150 & 300 & 350 & 300 & 250 & 150 \\
\hline
\end{tabular}

Table 5. The titer of IgG of lamb born from sheep vaccinated with MP12 vaccine using Elisa technique.

\begin{tabular}{|c|c|c|c|c|c|c|c|}
\hline \multirow{2}{*}{ Lamb No. } & \multicolumn{7}{|c|}{ Time / days post-colosrum ingestion } \\
\cline { 2 - 8 } & before & 2 & 30 & 60 & 90 & 120 & 150 \\
\hline 1 & -ve & 800 & 800 & 400 & 200 & 100 & $>50$ \\
\hline 2 & -ve & 400 & 400 & 200 & 100 & 50 & $>50$ \\
\hline 3 & -ve & 400 & 400 & 100 & 100 & 50 & $>50$ \\
\hline 4 & -ve & 800 & 800 & 400 & 200 & 50 & $>50$ \\
\hline Mean & -ve & 600 & 600 & 275 & 150 & 62.5 & $>50$ \\
\hline
\end{tabular}

Clinical observations of all sheep groups vaccinated with MP12 RVF vaccine were shown in Table 1. The results revealed that one sheep out of 18 ones on $1^{\text {st }}$ and $2^{\text {nd }}$ DPV had diarrhea. Six sheep In (G1) recorded febrile response $\left(40-40.9^{\circ} \mathrm{C}\right.$, which lasted for 2 days and returned to normal body temperature. These results 
agreed with Morrill et. al. (1991) who recorded that pyrexia was observed in some lambs vaccinated with MP12 RVF vaccine for short duration post-vaccination.

Pregnant ewes group (G2) showed no elevation of body temperature and delivered healthy lambs after 100 days post-vaccination. Results of RT- PCR for RVF virus detection in vaccinated sheep groups (G1 \&G2) on zero day were negative, while, on $2^{\text {nd }}$ day post-vaccination, it was found that 15 out of 18 serum samples were positive. This finding explains the short duration of vireamia (2 days).

Pregnant ewes (G2) showed no abortion and delivered lambs without abnormalities and remained healthy up to 4 months of age. These results agreed with Baskervillee et. al. (1992) who reported that abortion is not common sequel after vaccination of pregnant ewes with live attenuated Smithburn RVF vaccine.

The immune response following vaccination of sheep with MP12 vaccine was carried out using ELISA technique.

Table 2 showed that IgM antibodies were detected on 7 DPV in vaccinated sheep reaching the peak level on 14 DPV and persisted up to 150 DPV in all animals with a titer which ranged from 100 - 800 ELISA. This result agreed with Hassan et. al. (2009) who reported that IgM titer reached its peak after 14 days post-vaccination (DPV) of calves vaccinated with MP12 vaccine, while this titer decreased till 28 DPV.

Tables 3 \& 4 demonstrated that IgG antibodies were detected on 7DPV, reaching the peak on 60 DPV and persisted up to 360 DPV in all animals, with a titer which ranged from 100 - 800 ELISA. These results agreed with Hassan et. al. (2001).

Table 5 declared immunity of lambs born to MP12 vaccinated pregnant ewes which were negative at birth before ingestion of colosrum, then, increased to 600 ELISA titer after colosrum ingestion till 30 days, then, decreased reaching 62.5 at 120 days. This result agreed with Morril et. al. (1987) who found that lambs born to MP12 vaccinated pregnant ewes had antibody level less than 1:10 antibody titer at birth, and increased to 1: 80 after ingestion of colosrum.

The obtained results indicated that single dose of MP12 RVF vaccine is immunogenic and safe for non-pregnant and pregnant ewes. The duration of immunity was recorded up to one year post-vaccination. Lambs protective antibodies were detected after colosrum ingestion, and persisted up to three months proving the efficiency of the vaccine to protect lambs against RVF virus infection. 


\section{REFERENCES}

1. Baskerville, A., K. A. Hubbard and J. R. Stephanson. 1992. Comparison of the pathogenicity for pregnant sheep of Rift Valley Fever $\mathrm{V}$ and live attenuated vaccine.Res. Vet. Sci., 52 (3) : $307-311$.

2. Caplen, H., C. J. Peters and D. H. Bishop. 1985. Mutagen-directed attenuation of Rift Valley fever virus as a method for vaccine development. J. Gen. Virol., 66:2271-7.

3. Elian, K. A. and B. Botros. 1997. Production and evaluation of RVF diagnostic antigens by ELISA technique.3rd Arab Vet. Med. Cong. March 22-26,1997 Cairo.

4. Hassan, K.Z, K. A. Elian and M. M. Taha. 2001. Some studies on sheep vaccinated with smithburn attenuated Rift Valley Fever vaccine. Egypt .J.Agr.Res.,79 (3) :1141-1149.

5. Hassan, K. E. Z., A. M Ibrahim, A. S.Taradi, K.Elian, A. M. H. Azab, H. ElMohamady, and A. K.Soliman. 2009 .The effect of Rift Valley Fever vaccines on the release of interferon and immunoglbulin - $M$ in vaccinated cattle Alex. J. Vet. Sci. 28 (1) 110-2047.

6. Meegan, JM. 1979. The Rift Valley fever epizootic in Egypt 1977-78. 1. Description of the epizootic and virological studies. Trans. R. Soc. Trop. Med. Hyg.,73: 618623.

7. Morb IMortal Wkly (MMWR). 1994. International Notes: Rift Valley Fever - Egypt, 1993. MMWR Morb Mortal Wkly Rep.,43:693,699-700.

8. Morb Mortal Wkly MMWR. 2000.Outbreak of Rift Valley Fever -- Yemen August-October 2000. MMWR Morb Mortal Wkly Rep.,49:1065-1066.

9. Morril, JC., G. B., Jenning, H. Caplen, M. Turell, A. J. Johnson and C. J. Peters. 1987. pathogenicity and immunogenicity of a mutagen-attenuated Rift valley Fever immunogen in pregnant ewes. Am. J. Vet. Res., 78(7):1042 -1047.

10. Morrill, J. C., L. Carpenter, D. Taylor, H. H. Ramsburg, J. Quance and C. J. Peters. 1991. Further evaluation of a mutagen-attenuated Rift Valley fever vaccine in sheep. Vaccine, 9(1):35-41.

11. Office of International Epizootics (OIE). 2008. Manual of Diagnostic tests and vaccines for terrestrial Animals.6th Ed, Vol. 1: 323 - 333.

12. Office of International Epizootics (OIE). 1989. Manual of recommended diagnostic techniques and requirements for biological products of lists $A$ and $B$ diseases.International Committee Vol. 2 P. 1/8 - 8/8. 
13. Paweska,J.T., F.J. Burt and R. Swanepoel. 2005. Validation of IgG-sandwich and IgM-capture ELISA for the detection of antibody to Rift Valley fever virus in human.J. Virol. Methods, 124 (1-2) : 173-81.

14. Sall, A. A., E. A. Macondo, O. K. Sène, M. Diagne, R. Sylla, M. Mondo, L. Girault, L. Marrama, A. Spiegel, M. Diallo, M. Bouloy and C. Mathiot. 2002. Use of Reverse Transcriptase PCR in Early Diagnosis of Rift Valley Fever. American Society for Microbiology, Clinical and Diagnostic Laboratory Immunology, p. 713715, Vol. 9, No. 3.

15. World Health Organization (WHO). 2007. Rift Valley Fever WHO Media centre, Fact sheet $\mathrm{N}^{\circ} 207$. 


\section{تأثير لقاح حمى الوادي المتصدع المستضعف MP12 علي الأغنام}

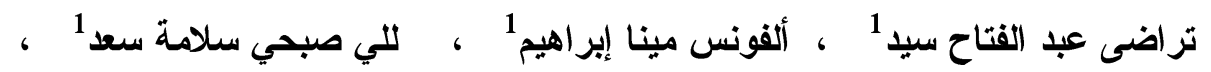
تيمور مرقص نصيف 1 ، عاطف كامل سليمان 2

1 معهد بحوث الأمصال واللقاحات البيطرية - مركز البحوث النرراعبة - وزارة النزراعة - الدقى - الجبزة NAMRU 2

تم دراسة الأعر اض المرضية والإستجابة المناعية للنعاج العشار وغير العشار المحصنة

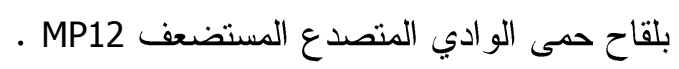

أظهرت النعاج الغير عشار إرتفاعاً طفيفاً في درجات الحرارة التي استمرت لمدة يومين

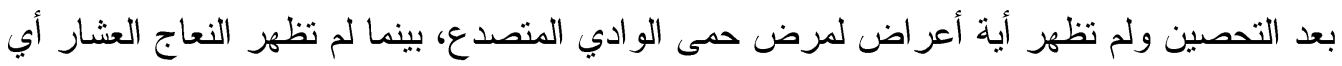
إرتفاع في درجات الحر ارة أو إجهاض وتم و لادة حملان طبيعية.

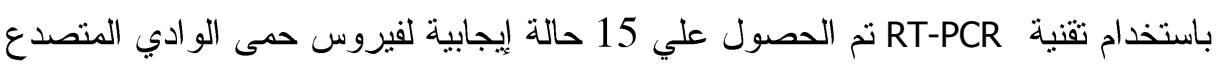
من 18 عينة مصل في اليوم الثاني بعد التحصين. وباجر اء إختبار الإليز اظهرت الأجسام المناعية IgM في اليوم السابع واستمرت حتى 150 يوماً بقوة عيارية من 100 من 100 - 800 ، بينما ظهرت الأجسام المناعية المة IgG في اليوم السابع بعد التحصين واستمرت حتى 360 يوماً ، أما بالنسبة

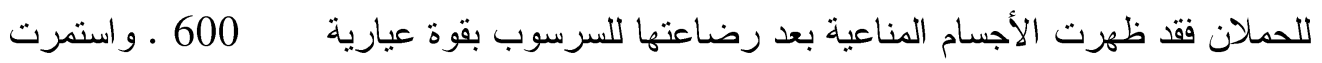
المناعية الأمية لمدة 4 شهور.

من النتائج السابقة يتضح أن لقاح حمى الو ادي المتصدع المستضعف MP12 آمن وفعال لتحصين الأغنام. 